\title{
Reflexiones sobre una propuesta formativa de profesores de Español como Lengua Extranjera (ELE) en Chile*
}

\author{
Manuel Rubio** \\ Raquel Rubio*** \\ Néstor Singer****
}

\begin{abstract}
Resumen
En este artículo se sistematiza la reflexión efectuada por un equipo docente de la Universidad de Santiago de Chile en el proceso de configurar una propuesta formativa para futuros enseñantes de ELE. El diseño del curso se centra en la didáctica concebida desde un enfoque que combina lo sociocomunicativo y la práctica reflexiva. La intencionalidad es desarrollar el sentido de plausibilidad en los participantes para que aprendan a considerar la clase de ELE como un proceso situado y único que demanda aprender a teorizar desde la práctica y postular el diseño de situaciones de aprendizaje como hipótesis de trabajo que deben ser contrastadas y reelaboradas constantemente.
\end{abstract}

Palabras clave: formación docente, español como lengua extranjera, diseño curricular.

\section{Reflecting on a Proposal for Educating Teachers of Spanish as a Foreign Language in Chile}

\begin{abstract}
This article conceptualises the reflections of a research team at the Universidad de Santiago de Chile during the process of designing of a teacher training proposal for future teachers of Spanish as a foreign Language (SFL). Such proposal considered didactics, understood as an approach which combines a socio-communicative stance and the reflective practice, as the main curricular axis. The proposal aims to develop a sense of plausibility in the trainees, so that they learn to consider teaching SFL as a unique process which requires them to learn to theorise from the teaching practice and plan learning situations as working hypothesis to be contrasted and reshaped constantly.
\end{abstract}

Keywords: Teacher education, Spanish as a foreign language, Curriculum design.

\footnotetext{
* Estudio adscrito al proyecto de diseño curricular del Diplomado en ELE, Departamento de Lingüística y Literatura de la Facultad de Humanidades.

** Chileno. Doctor en Lingüística por la Pontificia Universidad Católica de Valparaíso. Académico de la Universidad de Santiago de Chile, Santiago, Chile.manuel.rubio@usach.cl

*** Española. Magíster y Candidata a Doctora en Lingüística por la Universidad Autónoma de Madrid. Académica de la Universidad de Santiago de Chile, Santiago, Chile. raquel.rubio@usach.cl

**** Chileno. MA TESOL por The University of Manchester. Académico de la Universidad de Santiago de Chile, Santiago, Chile. nestor.singer@usach.cl
} 


\section{Introducción}

El español es una de las lenguas con mayor número de hablantes nativos y con un alto grado de influencia en el mundo de la cultura, las ciencias y las artes. Actualmente, se la considera la segunda lengua internacional de comunicación (Torres Torres, 2013). En concreto, el 7.6\% de la población mundial es hispanohablante y son más de 21 millones los estudiantes extranjeros que aprenden español tanto en sus países de origen como en algún país de habla hispana. En efecto, "por razones demográficas, el porcentaje de población mundial que habla español está aumentando (Instituto Cervantes, 2018), lo cual permite asegurar que una gran parte de la población se entenderá en español en las décadas venideras.

La demanda por aprender español y el potencial de futuros aprendices plantea la necesidad de formar docentes que cuenten con las herramientas teóricas, curriculares, didácticas y evaluativas para asegurar un buen desempeño. Actualmente, según datos del Instituto Cervantes (2018), la oferta de cursos de español se ha centrado en Norteamérica (Estados Unidos, Canadá y México) y la Unión Europea (especialmente España). En Chile, gradualmente comienzan a emerger las ofertas de cursos de español para extranjeros, aunque son pocas las instituciones que ofrecen cursos de especialización para profesores en esta área.

Las investigaciones recientes sobre enseñanza de lenguas extranjeras indican que la dinámica del aula debe posicionar al aprendiz como agente protagónico que moviliza sus pensamientos y emociones con el fin de gestionar la interacción con otros, dándole un uso significativo a lo aprendido (Cotterall, 2008; Benson, 2003, 2001; Little, 2004; Holliday, 2003). Por otra parte, se enfatiza que el aprendizaje de una lengua extranjera conlleva una modificación de aspectos ligados a la identidad, la visión de mundo, al sentido de pertenencia y a las prácticas sociales de interacción en un creciente contexto intercultural (Hecht, Warren, Jung \& Krieger, 2005; Johnson, 2004; Orbe \& Spellers, 2005).

Considerando lo anterior, se hace necesaria la articulación de programas de formación docente que habiliten a hablantes competentes para la enseñanza de la lengua y generar condiciones para un tipo de investigación centrada en la práctica pedagógica. Este desafío ha 
sido asumido por el Departamento de Lingüística y Literatura de la Universidad de Santiago de Chile, al interior del cual se constituyó un equipo enfocado a la enseñanza del español como lengua extranjera y al levantamiento de una línea de investigación en esta área.

Congruentemente, una de las primeras labores del mencionado equipo se orientó al diseño de un diplomado que habilitara a profesionales con formación lingüística para desempeñarse en la enseñanza de ELE. Afrontando dicho desafío, una de las primeras interrogantes abordadas se refiere a las características del proceso pedagógico, entendiendo que este se realiza con personas que tienen una formación previa, algunas experiencias en aula y que son adultos. Dicho cuestionamiento implica también plantearse una postura respecto de lo que es enseñar y el rol del enseñante, los desempeños esperados, las dificultades que implica enseñar una lengua a extranjeros, los saberes imprescindibles y la apropiación de dichos saberes.

El propósito de este artículo es sistematizar la reflexión efectuada por el equipo de ELE de la USACH en el proceso de configurar una propuesta formativa para futuros enseñantes de ELE. Compartir dicha reflexión puede constituirse en un aporte para otros equipos que deseen diseñar e implementar programas de formación de enseñantes de una lengua extranjera y es un primer paso para avanzar en la construcción de conocimientos sobre la enseñanza del español como lengua extranjera en esta universidad.

Cabe indicar que el artículo se organiza en tres apartados: el proceso de formación docente, la formación de profesores para enseñar una lengua a extranjeros y la racionalidad pedagógica en un programa de formación de enseñantes de ELE. El primer apartado plantea un marco general para comprender la formación docente dirigida a profesionales que cuentan con experiencias y conocimiento previo sobre la enseñanza y el aprendizaje; la segunda alude a principios teórico-prácticos orientados a la reconfiguración tanto de las representaciones como de la práctica pedagógica de los profesores en formación; finalmente, el tercero corresponde a la descripción del diseño del curso explicitando los principios en los cuales se asienta.

Finalmente, esta propuesta es novedosa en el campo de formación docente en ELE, ya que no se centra en los niveles de análisis lingüísticos (fonética, gramática, semántica y pragmática), sino que los aborda en forma integrada desde la perspectiva de la práctica docente. 


\section{El proceso de formación docente}

El proceso de formación docente para la enseñanza de ELE en un periodo intensivo requiere de ciertos requisitos. En relación con los participantes, dos son los fundamentales: contar con una formación lingüística previa y evidenciar un manejo competente de la lengua. Desde el punto de vista de la enseñanza y el aprendizaje, el proceso formativo debe centrarse en la didáctica concebida desde un enfoque que combina lo sociocomunicativo (Fernández Enguita, 1996; Medina \& Mata, 2002) y la práctica reflexiva (Schön, 1992; Zeichner, 1993; Zeichner \& Liston 2014; Richards \& Lockhart, 1996).

Desde una perspectiva sociocomunicativa, la didáctica es una interacción de carácter comunicativo que involucra una dimensión proposicional ligada a los contenidos verbalizados en el proceso y un vínculo relacional entre los participantes que se realiza en un contexto que abarca desde los aspectos inmediatos de la situación hasta la dimensión cultural en la que dicho proceso se inscribe.

Desde la práctica reflexiva, la didáctica es una interacción centrada en el despliegue de un pensamiento racional para el análisis de la experiencia propia o ajena con el fin de transformar creencias, actitudes, concepciones y saberes. El propósito es que los docentes trasciendan la lógica de la aplicación de planteamientos teóricos y soluciones estandarizadas determinados externamente y construyan un saber profesional que posibilite filtrar los planteamientos teóricos y curriculares así como reelaborar y proponer situaciones de aprendizajes coherentes con las características de los estudiantes y las demandas del entorno.

La articulación entre lo sociocomunicativo y la práctica reflexiva supone la creación de un clima emocional en el aula y el diseño de situaciones de aprendizaje que potencien el diálogo cooperativo y el compromiso de los participantes para que objetiven sus experiencias y saberes, los problematicen y los reestructuren.

En esta línea, la interacción pedagógica implica la concurrencia de tres elementos fundamentales que interactúan en un contexto cultural que sirve de condicionamiento y que se va reconstruyendo, ampliando o restringiendo las posibilidades de encuentro e interacción entre los participantes. Los tres elementos son el profesor, el alumnado y el contenido curricular. 
En el proceso de formación de profesores, proponemos que la vivencia de este enfoque sociocumunicativo y práctico reflexivo sirva como modelaje para que los futuros enseñantes de ELE configuren estrategias para el diseño de situaciones de aprendizaje potenciadoras y que se apropien de la idea de que al profesor le corresponde realizar dos tipos de actividades: el establecimiento de relaciones y la pedagogización del contenido.

El profesor debe configurar un estilo pedagógico que considere la dimensión relacional del proceso comunicativo y aprender a generar vínculos y un ambiente de confianza que potencie la confianza de los estudiantes para comprometerse en el proceso de aprendizaje (Arnold, 2000; Rodríguez-Pérez, 2012; Pizarro, 2013; Iglesias Casal, 2016). El vínculo pedagógico implica que el docente exprese una comprensión empática para aceptar las diferencias culturales. En esta perspectiva, el clima al interior de la sala de clases es un rasgo sobresaliente, puesto que hay climas productivos que animan el aprendizaje versus otro tipo de climas que favorecen la abulia o el temor. ¿Qué características tienen aquellos climas que motivan el aprendizaje? Fuera de los contenidos vinculados al conocimiento de la lengua, debieran contemplarse habilidades y estrategias para promover la convivencia y aceptar las diferencias culturales, apertura para abordar un tema desde diversas perspectivas, manejar la estructura de la clase de tal manera que esta no ahogue las posibilidades de expresión. En este sentido, una perspectiva crítica de la propia cultura es un factor clave para no intentar posicionar ciertos estilos, principios y valores como los únicamente válidos. También es necesario comprender que, en la medida que se aprende una lengua extranjera, no solo se aprende un léxico determinado y reglas gramaticales, sino pautas sociales de interacción y contenido simbólico en torno a cómo se concibe y actúa en el mundo. Esto implica una posible disonancia en los aprendices de español, por lo cual el cual el profesor en formación debe internalizar que ellos, al aprender una lengua distinta de la materna, desarrollan también un proceso de extrañamiento de su propia cultura (Hecht, Warren, Jung \& Krieger, 2005; Johnson, 2004). Dicho extrañamiento puede ser consciente o inconsciente, asumido o resistido y, por lo tanto, constituirse en una suerte de obstáculo cultural que es necesario tener presente en el mantenimiento y desarrollo del vínculo relacional.

El profesor debe enfocarse en la dimensión proposicional del proceso mediante la pedagogización del contenido, proceso que supone la 
selección, organización y graduación de lo que se espera que el estudiante aprenda. Dicho proceso es complejo, pues supone la articulación de actividades de aprendizaje que signifiquen un estímulo y que estén en el ámbito de posibilidades del grupo de estudiantes con los cuales se trabaja. Desde esta perspectiva, el profesor no puede asumir una postura mecánica o lineal del aprendizaje, sino que debe evidenciar una apertura para reflexionar sobre el proceso, sus fortalezas y debilidades, a modo de instalar el proceso en el ámbito de la zona de desarrollo potencial de aprendizaje de los estudiantes (Kozulin, Gindis, Ageyev \& Miller, 2003). En este sentido, un enfoque flexible y emergente puede ser relevante para favorecer aprendizajes significativos y, por tanto, perdurables.

Dicha pedagogización está asociada al diseño de situaciones de aprendizaje para que los estudiantes se involucren y puedan avanzar hacia el logro de los resultados de aprendizaje. Este diseño no puede entenderse como algo cerrado antes de la interacción, sino que se realiza y modifica en la propia interacción. En esta línea, la clase no está predefinida, sino que es una interacción emergente que implica un trabajo conjunto entre el docente y el alumnado.

El proceso de formación de enseñantes que proponemos apunta a que los participantes reconozcan que una clase de enseñanza de una lengua debe ser entendida como un encuentro intercultural que supone un diálogo que apunta a la reestructuración de representaciones sociales y de pautas de interacción. En esta línea, es fundamental concebir dicho encuentro como un proceso de ampliación del mundo de los sujetos involucrados en el proceso de enseñanza. El aprendizaje de la lengua meta supone cambios de paradigmas. Por ello, resulta clave que el profesor genere las condiciones que posibiliten el tránsito de una cultura a la cultura de la lengua meta para que el aprendiz pueda insertarse en ella e interactuar con otros de manera asertiva.

En concreto, la formación docente en el ámbito de la enseñanza de una lengua apunta a tres tipos de saberes que se deben articular entre sí: el uso competente de la lengua, un saber metalingüístico y un saber pedagógico. En efecto, el docente que enseña una lengua debe ser un usuario competente de la misma. Dicha competencia involucra no solo hablar la lengua, sino también adquirir conciencia sobre los elementos culturales que condicionan dicho uso de acuerdo a diversos registros y situaciones de comunicación: qué es posible y deseable decir, a quiénes, en qué situaciones concretas; qué se debe evitar decir, a quiénes y en qué 
condiciones. Por su parte, el saber metalingüístico es fundamental para comprender el funcionamiento de la lengua e identificar fenómenos y estructuras que sean necesarios enseñar.

En relación con el saber pedagógico, se destaca la práctica reflexiva que posibilita comprender, observar y reflexionar críticamente sobre el proceso de enseñanza y aprendizaje; la capacidad de aprender de la propia práctica docente construyendo un saber validado en el quehacer; asimismo, el docente aprende a autoobservarse para adquirir mayor consciencia de las representaciones que porta y observar las representaciones que, a su vez, poseen sus estudiantes sobre los diversos aspectos involucrados en el proceso de enseñanza y aprendizaje. En esta línea, el saber pedagógico corresponde a una síntesis personal que articula teoría, experiencia y representaciones que permite darle sentido al quehacer, comprender las dificultades y diseñar estrategias compensatorias.

En el proceso de formación de profesores, es necesario considerar el saber pedagógico que portan los futuros enseñantes. En otras palabras, es necesario movilizar, explicitar y problematizar las concepciones que el docente posee sobre los diferentes elementos presentes en el proceso de aprendizaje de una lengua. Esto es una actividad compleja, pues no necesariamente los contenidos que configuran una representación son elementos conscientes. Incluso puede ser que un sujeto no se dé cuenta del trasfondo en que se configura una opinión expresada explícitamente. El profesor en formación ha construido un conocimiento de sentido común que se acepta muchas veces como coherente e incuestionable, puesto que permite explicar y comprender muchas situaciones vinculadas al proceso de enseñanza y aprendizaje.

Explicitar dicho conocimiento, descubrir sus potencialidades y debilidades significa convertirlo en objeto de reflexión, en un problema que es necesario descubrir y analizar. Eso significa que es necesario expresar ese saber en enunciados concretos que, mediante un proceso de diálogo guiado a través de preguntas, permita develar los supuestos implícitos y el marco de referencia desde el cual se expresa. La pregunta que indaga, que permite la formulación de nuevos enunciados para compartir con otros y que devela dichos contenidos, es crucial. Esta no es una pregunta que impone una mirada, sino una pregunta que invita a remirar lo dicho, a tensionarlo, a generar disonancias, que permitan tomar conciencia de los elementos de trasfondo presentes en ellos. 
Este proceso de problematización vivido en situaciones de diálogo cooperativo con otros es lo que permite incorporar nuevas miradas, otros puntos de vista, experiencias diferentes y, por lo tanto, avanzar en su reestructuración.

Además, dicho proceso de develamiento, permite introyectar un modo de ser en la acción, es decir, aprender a evaluar diferentes perspectivas y opciones al momento de tomar decisiones y reflexionar sobre la práctica.

\section{La formación de profesores para enseñar una lengua a extranjeros}

La enseñanza de lenguas extranjeras ha experimentado una serie de cambios en las últimas décadas. Dichos cambios corresponden a diferentes paradigmas sobre la psicología del aprendizaje de una segunda lengua, pasando por la gramática universal de Chomsky (1959), la teoría del input y del monitor de Krashen (1982), la teoría del output de Swain (1985), para llevar finalmente a las teorías socioculturales y constructivistas.

Estos paradigmas han generado, a su vez, una serie de enfoques en la enseñanza de lengua que, $\operatorname{como}$ señala $\operatorname{Cook}(2003$, p. 30), "no ocurrieron al azar, sino que en respuesta a escenarios geopolíticos y valores y actitudes sociales cambiantes, así como también a cambios de tendencias en la lingüística" (nuestra traducción). Dichos cambios promovieron la creación de métodos que competían entre sí en la búsqueda de ser el único y mejor. A pesar de las diferencias entre ellos, todos tenían una característica común: proponer una serie de principios únicos que determinan si ocurrirá aprendizaje o no (Nunan, 1991). Así es como encontramos el método tradicional, directo, audiolingual, respuesta física total, comunidad de aprendizaje de idiomas, comunicativo y enfoque por tareas, entre muchos otros (Hall, 2011; Larsen-Freeman, 2000).

En este escenario, Prahbu (1990) sostiene que no existe un mejor método, sino que más bien todo enfoque está supeditado a las representaciones del profesor en cuanto a qué tan viable o pertinente es un determinado paradigma, método o técnica para el contexto en el cual se desenvuelve. Prahbu llama a esta capacidad 'sentido de plausibilidad'. De manera similar, Kumaravadivelu (2006) señala que hoy por hoy nos encontramos en la era de posmétodo y establece tres principios clave para comprenderlo: particularidad, posibilidad y practicidad. La particularidad está relacionada con que los profesores actúan en 
contextos únicos con alumnos de características lingüísticas, culturales y sociales específicas; la posibilidad involucra insertar dentro del aula los aspecto sociopolíticos de los alumnos para promover la formación de identidades y transformación social; y la practicidad se relaciona con la capacidad de los profesores para teorizar desde su práctica docente en búsqueda de determinar qué es lo mejor para su contexto.

Este último punto es el resultado de varios procesos complejos cognitivos y metacognitivos en los que los profesores reconfiguran sus representaciones sobre la enseñanza y aprendizaje y, a su vez, su práctica y diversos tipos de conocimiento docente. En el siguiente apartado analizaremos los diferentes saberes del profesor, su interacción con las habilidades y rol en el cambio de representaciones sobre la enseñanza y aprendizaje de una lengua extranjera.

\subsection{Conocimientos del profesor}

El profesor de lengua debe tener un mínimo de conocimientos para poder articular los elementos socioculturales del aula de manera armoniosa $\mathrm{y}$, en consecuencia, desempeñarse de manera efectiva en su contexto. Freeman (1989) propone un modelo descriptivo para la enseñanza en donde reconoce cuatro constituyentes principales. El primer elemento se denomina conocimiento, vale decir, el qué enseñamos. No obstante, este también puede comprender una serie de otros conocimientos, tales como conocimiento de estudiantes y del contexto donde enseñan. A estos tipos, Shulman (1986) agrega el conocimiento pedagógico general, del contenido pedagógico propio de la disciplina y del currículum. Estos últimos tres tipos de conocimiento se relacionan con el segundo constituyente del modelo: habilidades. Estas involucran el universo de los métodos de enseñanza para una segunda lengua, técnicas, actividades, tareas. También se relaciona con el uso de materiales y herramientas disponibles. En otras palabras, esta dimensión abarca el cómo se enseña. El tercer elemento de la propuesta se denomina actitud, definida como una postura respecto a uno mismo como profesor, a las actividades y a otros. Dicha postura vincula las dinámicas internas con el actuar docente en el aula. En otras palabras, corresponde a las percepciones o representaciones que el profesor tiene sobre sí mismo y el proceso de enseñanza y aprendizaje.

En relación a esto último, Borg (2003) señala que las representaciones sociales son resultado de las experiencias previas tanto de los profesores 
como de los alumnos, en los programas de formación profesional y en la práctica profesional. Esto quiere decir que las representaciones surgen desde la interacción de las experiencias, la teoría y la práctica, por lo que subyace por qué hacemos lo que hacemos. La evolución y desarrollo de dichas representaciones es un proceso complejo; la literatura sobre el impacto que tienen cursos de formación docente en el desarrollo de la cognición docente muestra dos caras opuestas. Aquellas investigaciones que se han centrado en los contenidos de dichas representaciones han demostrado que estos cursos no son del todo efectivos para lograr un cambio significativo en las representaciones docentes. No obstante, aquellos estudios que se han enfocado en los procesos y estructura del desarrollo cognitivo muestran resultados totalmente contrarios (Ávalos, 2007, 2011).

Lo anterior sugiere que existe otro elemento que informa y gatilla los distintos procesos de desarrollo de las representaciones de los profesores. Dicho elemento corresponde al cuarto constituyente del modelo de Freeman: la conciencia, definida como 'la capacidad de reconocer y monitorear la atención que uno le está dando o le ha dado a algo' (1989, p. 33) (nuestra traducción). Por ejemplo, un profesor puede corregir los errores de sus estudiante mediante una técnica específica, pero si en algún momento se da cuenta de que la técnica o proceso de evaluación no es el adecuado, entonces este descubrimiento por parte del docente puede desencadenar un cambio en las representaciones y en el conocimiento que el profesor posee en cuanto a la corrección de errores en la sala de clases. En otras palabras, es este elemento el que permite a los profesores cuestionar por qué realizan algo de la forma que lo hacen y, en consecuencia, podría alterar el cómo y qué enseñan.

Los cambios en las percepciones docentes surgen como resultado de este estado de conciencia y de un proceso de reflexión por parte del docente. Bengtsson (1995) la define como la capacidad humana de volverse hacia sí mismo para descubrir ciertos aspectos sin ayuda de terceros (reflexión de uno mismo). Lo anterior implica una introspección en que el profesor se desdobla para tomar una actitud más crítica y objetiva ante su práctica docente (reflexión como pensamiento) con el fin de comprender qué hace, cómo lo hace y porqué lo hace (reflexión como propio entendimiento). Este proceso puede llevar a cambios tangibles como, por ejemplo, el cambio de una determinada metodología en el 
aula, así como también a cambios cognitivos en torno a la enseñanza de una lengua.

La Figura 1 resume esto en una adaptación del modelo de Freeman (1989) y posiciona la reflexión como proceso cognitivo, como el elemento capacitador de la práctica docente y como factor reestructurador de las percepciones sociales de los profesores.

Figura 1. Adaptación del modelo descriptivo de enseñanza de Freeman (1989).

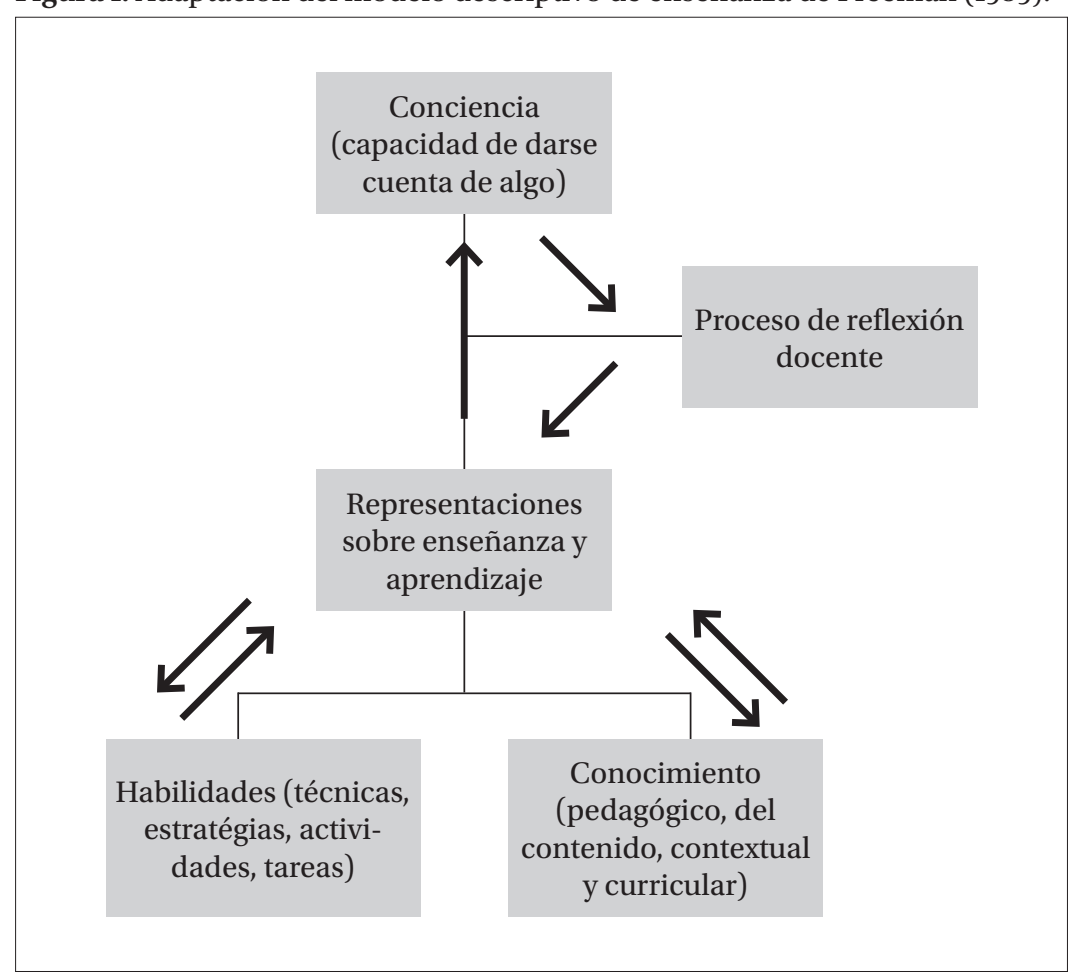

\subsection{Hacer explícito lo tácito: formando profesores}

De los elementos anteriores, podemos concluir que, para que la reflexión conduzca a una restructuración de las representaciones del profesor, este debe estar consciente de las diferentes situaciones que se producen dentro del universo de la sala de clases.

El dilema, entonces, para la capacitación de profesores, es cómo lograr desarrollar la capacidad reflexiva del profesor en formación que le permita una selección de habilidades coherentes al contexto, justificada en un conocimiento pedagógico sólido en armonía con sus 
representaciones. Lo anterior es posible si el contraste de los contenidos presentados y discutidos en el curso logra emular la dicotomía entre el conocimiento teórico y la experiencia práctica en el aula para lograr el proceso de toma de conciencia y así desencadenar la reflexión. Dichas situaciones deben además incluir una secuencia que permita el establecimiento de un diálogo constructivo y cooperativo entre los participantes y el individuo que potencie la restructuración de los saberes docentes, vale decir, una metodología que tenga como eje articulador la didáctica sociocomunicativa. Esta se entiende como un escenario complejo de relaciones entre profesores y alumnos, los símbolos utilizados por los mismos y, lo más importante, la valoración que estos les asignan y su interpretación de las percepciones subyacentes (Medina \& Mata, 2002).

En este proceso de discusión colectiva para negociar significados, se puede lograr que los profesores en formación se den cuenta y verbalicen explícitamente sus representaciones sobre la enseñanza de lenguas en relación a la teoría y práctica presentada, así como también a lo expuesto y elaborado con el curso. En otras palabras, según Shulman (1988, p. 33) 'hace[n] explícito lo tácito' (nuestra traducción).

No obstante, el proceso de construcción de significados que llevan a la explicitación se produce cuando el proceso es lo suficientemente significativo para el profesor. Kolb (1984) señala las características del aprendizaje experimental, las cuales fueron adaptadas en esta propuesta como una serie de principios para el diseño de secuencias didáctica con el fin de lograr traer a la superficie las representaciones docentes: la reflexión 1) debe ser entendida como proceso de negociación interna o colectiva y no como un producto, 2) es un proceso continuo y basado en la experiencia previa o emulada, 3) es un proceso holístico de adaptación al mundo real. Estos tres principios promueven el desarrollo de una capacidad permanente de cuestionamiento de conocimiento y habilidades, ya que, para poder generar estrategias que dan respuestas al mundo real de la enseñanza de idiomas, los profesores deben encontrar posibles soluciones a conflictos y tensiones conceptuales, procedimentales y actitudinales. Lo anterior hace que los alumnos personalicen la experiencia de las diferentes actividades y de esta forma, sea un aprendizaje significativo con mayores probabilidades de causar una restructuración en las representaciones docentes. 
Para realizar dicho proceso, el análisis práctico de situaciones reales del aula se posiciona como objeto generador de diálogo con el fin de develar la teoría subyacente, problematizar dicha teoría con la practicidad del aula y, quizás más importante, con las representaciones que los profesores traen al aula. De esta forma, las representaciones de los profesores actúan como un filtro de los conocimientos prácticos y teóricos discutidos por el grupo y estos, a su vez, permiten la restructuración, evaluación o cambio de las percepciones docentes que originalmente trajeron los otros participantes al aula.

Dichas situaciones incluyen el análisis de materiales de enseñanza, análisis de casos y análisis de problemas como posibles estrategias para potenciar el diálogo colectivo. En el caso de los primeros, estos apuntan a que los profesores desarrollen habilidades para la selección de materiales y contenidos al explorar diversos tipos de textos utilizados hoy en día, ya sean publicados por editoriales o diseñados por profesores participantes del curso de manera individual. Esto permite al profesor indagar en los aspectos teóricos de la enseñanza de lengua mediante una exploración de los enfoques subyacentes en los materiales y determinar qué es útil o no en un determinado contexto. Lo anterior potencia el sentido de plausibilidad planteado por Prabhu (1990).

El análisis de caso es relevante ya que, como señala Díaz Barriga (2006), permite a los alumnos conocer la realidad del aula sin necesariamente estar en ella, y desarrollar vínculos de identificación y empatía. Los casos permiten problematizar los procesos de enseñanza aprendizaje, así como también generar controversia en torno a las estrategias de enseñanza utilizados por profesores. Esto último ayuda a que los docentes comprendan de mejor forma los encuadres teóricos y, en consecuencia, potencia la vinculación de los mismos con el currículum en la enseñanza de lenguas. El análisis de problemas, por su parte, potencia las mismas áreas, con la diferencia de que, al tratarse de casos de la realidad del aula, estos tienen mayor impacto por su relevancia para la práctica docente $y$, en consecuencia, mayor probabilidad que los profesores personifiquen la experiencia de aprendizaje.

Como resultado de estos tipos de actividades y tareas de aprendizaje, los profesores en formación irán desarrollando capacidades de pensamiento crítico y de reflexión en la acción, así como también adquirirán el conocimiento pedagógico. Además, les permitirá redefinir sus representaciones sobre la enseñanza de lenguas y, en consecuencia, 
desarrollar una identidad y postura crítica ante los diferentes fenómenos en el campo de la enseñanza y aprendizaje de lenguas extranjeras.

\subsection{Principios para la formación de profesores: reflexionar en la acción}

En síntesis, el hacer explícito lo tácito permite la reflexión y cuestionamiento de representaciones docentes, así como también potencia una toma de decisiones pedagógicas en momentos específicos. No obstante, estas decisiones no se establecen como un recetario descontextualizado de técnicas, sino como resultado de decisiones razonadas y de un deseo de perfeccionamiento constante de la práctica docente. En otras palabras, el fin último de hacer explícito lo tácito es la capacidad permanente y efectiva para que el profesor sea capaz de reflexionar en la acción.

En resumen, los principios fundamentales para la formación docente que inspiran nuestra propuesta son:

1. La interacción y la retroalimentación son los principios básicos que deben considerarse al momento de planificar, estructurar y evaluar una clase de idiomas.

2. El desarrollo de vínculos afectivos y la comprensión empática entre el profesor y el alumnado favorece un clima potenciador del aprendizaje y la configuración de un clima de confianza donde el error no está penalizado.

3. El profesor de lengua debe concebirse como un referente o modelo en el manejo de la lengua y en la sensibilidad frente a aspectos culturales para configurar la clase como un lugar de encuentro intercultural que valora la diferencia.

4. El foco de un programa de formación de profesores de lengua debe ser la didáctica entendida como la articulación entre una dimensión sociocomunicativa y una dimensión de la práctica reflexiva.

5. El proceso de reflexión docente solo puede activarse cuando estos logran darse cuenta de los eventos que ocurren en la sala de clases.

6. La reflexión docente debe concebirse como un proceso continuo y holístico que busca dar soluciones a problemas reales del aula.

7. Mediante una metodología que aborde el diálogo constructivo para hacer explícito lo tácito, los profesores tienen mayores posibilidades de personalizar su experiencia de aprendizaje y, en consecuencia, 
mayor posibilidad de restructurar sus representaciones sobre la enseñanza de lenguas.

8. El fin último de la capacidad reflexiva es la habilidad del profesor de tomar decisiones efectivas durante su desempeño docente, ya sea in situ o a priori, visualizando dichas decisiones como hipótesis de trabajo que constantemente deben ser contrastadas con la práctica pedagógica situada.

\section{Racionalidad pedagógica en un programa de formación de enseñantes de ELE}

El diseño de un curso de diplomado para la formación de profesores de ELE considera un periodo lectivo intensivo de 150 horas cronológicas, distribuidas en dos sesiones semanales de tres horas cada una durante ocho meses. Por tal motivo, el perfil de ingreso especifica como público objetivo a profesionales o licenciados que cuentan con formación en lingüística y que evidencian un manejo competente del español.

El foco del curso es la didáctica del español como lengua extranjera, concebida como un proceso de interacción comunicativa centrado en la práctica, que vincula reflexión y acción sobre los diversos componentes que integran el proceso de enseñanza y aprendizaje de una lengua. El propósito es que los profesores en formación configuren un saber profesional, es decir, una matriz de sentido fundada en conocimientos, habilidades y representaciones sobre la lengua, los aprendices, el contexto, el currículum, el rol docente, entre otros, que posibilite la toma de decisiones razonada para diseñar situaciones de aprendizaje potenciadora de la competencia comunicativa definida desde una perspectiva intercultural.

Congruentemente, la formación de profesores de ELE requiere de la vivencia de una práctica reflexiva constante, personal y dialogada dirigida a la toma de conciencia de aspectos concretos, explícitos y tácitos, del proceso de enseñanza y aprendizaje de la lengua que permita tomar decisiones acertadas y proponer soluciones adecuadas al contexto y a las necesidades de los aprendices. En concreto, el curso se orienta a la creación de condiciones para que los profesores en formación adquieran una apertura a los desafíos de la práctica mediante un proceso de reestructuración de sus representaciones y potenciación de su capacidad analítica para develar las concepciones presentes en diversas propuestas 
curriculares y en diferentes materiales didácticos. En este sentido, se busca que los participantes conciban el diseño didáctico como la elaboración de hipótesis de trabajo que requieren ser contrastadas en situaciones de aprendizaje situadas.

Para tal efecto, los pilares del curso de formación de profesores de ELE lo constituyen tres tipos de saberes. Estos son:

1. Saber sobre el español desde una perspectiva lingüística y discursiva, relevando las problemáticas del uso de esta lengua de acuerdo con diferentes géneros y registros, desde los más cotidiano y concreto hasta los más especializados y abstractos. En efecto, el proceso de enseñanza de una lengua extranjera no se enfoca en el conocimiento de dicha lengua como sistema abstracto viable de ser descrito de manera formal. Por el contrario, la idea es un enfoque funcional que incorpora factores del contexto situacional y cultural y el logro de diversos propósitos. En este sentido, se promueve una comprensión de la lengua como un sistema semiótico social que permite la construcción de significados ideacionales e interpersonales que exigen del hablante competente la necesidad de realizar opciones de significación en diferentes dimensiones (Halliday \& Matthiessen, 1999, 2004; Halliday 1998, 2003). Del mismo modo, el aula de ELE corresponde a un encuentro de sujetos situados socioculturalmente. La cultura es lo que dota de identidad a los sujetos y condiciona su actuar. Aprender una lengua es vivir un proceso de transición entre la propia cultura y la cultura de la lengua meta. Eso implica un proceso de extrañamiento de la propia cultura y el reconocimiento de otras posibilidades de pensar lo humano, de configurar lo social e interactuar bajo el respeto y la tolerancia hacia la diversidad.

2. Saber pedagógico de la enseñanza de una lengua extranjera, relacionado con modelos, estrategias y metodologías de enseñanza entendidos como un fenómeno complejo, cuya realización implica una toma de postura en torno a cómo los sujetos aprenden y dotan de significado su quehacer compartido con otros. Esto requiere de conocimiento instrumental por parte de los docentes en términos de cómo organizar situaciones de aprendizaje y reunir antecedentes que permitan juzgar las dificultades y avances que los aprendices de ELE pueden tener con el propósito de reorientar el proceso de enseñanza y aprendizaje (Iglesias Casal, 2016). En este sentido, la formulación de objetivos de aprendizaje, la selección de contenidos de lengua, el 
diseño de actividades y los procesos de evaluación que se configuren coherentemente entre sí constituyen un elemento central.

3. Saber convivir y actuar con otros para que el aula de ELE se asuma como un encuentro de sujetos diversos no solo socioculturalmente, sino también respecto de sus necesidades, motivaciones, actitudes y aptitudes para el aprendizaje. El profesor de ELE debe contar con los conocimientos necesarios para poder establecer un clima de aceptación y confianza que fomente la participación y la motivación hacia el aprendizaje, además del respeto y el reconocimiento hacia la diversidad para poder establecer situaciones de colaboración y aprendizaje efectivas, encaminadas a la construcción de significados compartidos (Arnold, 2000; Pizarro, 2013).

En congruencia con la opción por un enfoque sociocomunicativo y de la práctica reflexiva en la formación docente, el curso considera la articulación dialéctica entre teoría y práctica en un proceso de complejidad creciente. En otras palabras, el diseño curricular del curso apuesta por una concepción del aprendizaje en espiral, según la cual se retoman los mismos temas, pero con un nivel de complejidad mayor. Dicha concepción se ha representado gráficamente en la Figura 2.

Figura 2. Representación gráfica del modelo curricular para el diseño del curso de formación de profesores de ELE.

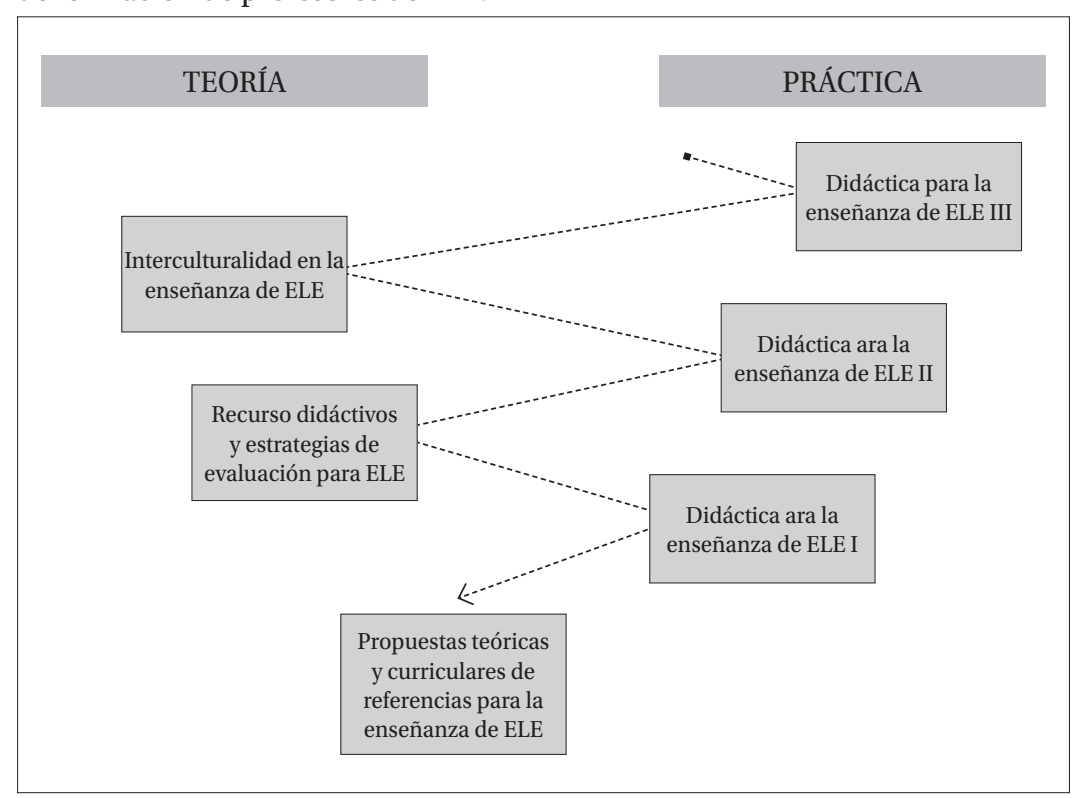


En el polo teórico, se parte por el análisis crítico de propuestas teóricas y curriculares para la enseñanza de ELE, tales como el Marco Común Europeo de Referencia para la Enseñanza de Lenguas, los Performance Descriptors for Language Learners del American Council on The Teaching of Foreign Language (ACTFL), el Plan Curricular del Instituto Cervantes y planteamientos teóricos de la lingüística aplicada a la enseñanza de lengua. Posteriormente, se continúa con elementos de planificación, didáctica y estrategias de evaluación para ELE. Finalmente, se consideran aspectos vinculados con la interculturalidad en la enseñanza de ELE.

En el polo práctico, se analizan y modelan estrategias didácticas para la enseñanza de ELE, se develan las teorías y principios subyacentes en materiales didácticos de diferentes instituciones y los propios diseñados por los participantes. Finalmente, se pasa al diseño de propuestas de situaciones de aprendizajes que respondan a un caso concreto.

Vale insistir en que el abordaje de tales contenidos teórico-prácticos no es lineal, sino que es necesario concebirlos como dimensiones fundamentales que constituyen los referentes permanentes del curso, por lo cual se vuelve a ellos y se los complejiza de acuerdo con demandas analíticas y procedimentales crecientemente más complejas. Dicha complejidad se relaciona con la progresión de los aprendizajes de los participantes, el debate emergente en el curso, el diseño de situaciones de aprendizaje para aprendices de español con diferentes niveles de dominio de la lengua, aprendices con diferentes situaciones existenciales y la incorporación de géneros discursivos especializados. Por ejemplo, las demandas analíticas y procedimentales con que se consideran dichos referentes son muy distintas si se les solicita a los profesores en formación que diseñen, por un lado, un proyecto pedagógico de intervención destinado a emigrantes haitianos, quienes se atienden en un consultorio de la periferia de Santiago, viven en situaciones de hacinamiento y acceden a actividades laborales relativamente informales, para que puedan acceder a una atención de salud de calidad; y, por otro, si se les solicita el diseño de una secuencia didáctica para estudiantes universitarios europeos que complementan su formación profesional en un programa especial de aprendizaje del español en nuestra universidad y que cuentan con medios económicos para acceder a condiciones favorables de alimentación y vivienda.

En esta concepción que articula dialécticamente teoría y práctica en un nivel creciente de complejidad, resulta esencial no solo el contenido, 
sino el proceso de enseñanza, entendido como la forma en que se construyen los diferentes tipos de saber que requiere el profesor en formación. Forma y contenido no pueden ser contradictorios entre sí, sino que deben vincularse coherentemente.

Concretamente, la forma de intencionar la reestructuración de representaciones y la construcción de saberes es mediante el análisis de casos, la reconstrucción lingüística de la experiencia propia y ajena, el análisis de materiales de enseñanza, la resolución de problemas, el diseño de nuevas propuestas didácticas. En otras palabras, el proceso de aprendizaje considera la problematización de la teoría y la práctica mediante el despliegue de un razonamiento colectivo centrado en la interrogación y el develamiento de supuestos implícitos.

\section{Conclusiones}

Múltiples son las demandas que surgen cuando se considera la creciente influencia del español en el mundo de la cultura, las ciencias y las artes. Una de ellas hace referencia a la necesidad de debatir en torno a la formación de profesores que enseñen esta lengua a extranjeros. Asumiendo dicho desafío, se han presentados los fundamentos en torno a los cuales se procedió a diseñar una propuesta de formación de profesores de ELE en la USACH.

La propuesta combina dos enfoques complementarios entre sí: el sociocomunicativo y el de la práctica reflexiva con la finalidad de generar las condiciones para que un grupo de profesores en formación construyan un saber profesional que les permita filtrar, reelaborar y proponer principios teóricos, planteamientos curriculares y situaciones de aprendizajes sensibles al contexto y características de diferentes tipos de aprendices de ELE. Al respecto, se destaca la concepción del aula de ELE como un lugar de interacción sociocultural que se configura dinámicamente y que plantea desafíos muchas veces difícil de prever.

Si bien dicha propuesta está centrada en la didáctica, no se apunta a la búsqueda de un mejor método, sino más bien a desarrollar el sentido de plausibilidad en los participantes para que aprendan a considerar la clase de ELE como un proceso situado y único que demanda aprender a teorizar desde la práctica y postular el diseño de situaciones de aprendizaje como hipótesis de trabajo que deben ser contrastadas y reelaboradas constantemente. 
Este artículo se centró en la descripción del diseño del curso. En consecuencia, un desafío a abordar en el futuro cercano es dar cuenta de la sistematización de su puesta en práctica, caracterizando las eventuales tensiones y fortalezas, la reestructuración de las representaciones de los profesores en formación y los eventuales aportes que se pueden realizar a las teorías de formación de profesores para la enseñanza del español como lengua extranjera.

\section{Referencias bibliográficas}

Arnold, J. B. (2000). La dimension afectiva en el aprendizaje de idiomas. Madrid: Cambridge University Press.

Ávalos, B. (2007). El desarrollo profesional continuo de los docentes: lo que nos dice la experiencia internacional y de la region latinoamericana. Pensamiento educativo 41(2), 77-99.

Ávalos, B. (2011). Teacher professional development in Teaching and Teacher Education over ten years. Teaching and Teacher Education, 27, 10-20.

Bengtsson, J. (1995). What is Reflection? On reflection in the teaching profession and teacher education. Teacher and Teaching: theory and practice, $1(1), 23-32$.

Benson, P. (2001). Autonomy in Language Learning. Harlow: Longman.

P. (2003). Learner Autonomy in the Classroom. En D. Nunan (Ed.), Practical English Language Teaching (pp. 289-308). New York: McGraw-Hill.

Borg, S. (2003). Teacher cognition in language teaching: A review of research on what language teachers think, know, believe, and do. Language Teaching, 36(2), 81-109.

Chomsky, N. (1959). Review of "Verbal Behaviour" by B. F. Skinner. Language, 35(1), 26-58.

Cook, G. (2003). Applied Linguistics. Oxford: Oxford University Press.

Cotterall, S. (2008). Autonomy and Good Language Learners. En C. Griffiths (Ed.), Lessons from Good Language Learners (pp. 110-120). Cambridge: Cambridge University Press.

Díaz Barriga, F. (2006). Enseñanza situada: Vínculo entre la escuela y la vida. Ciudad de México: McGraw Hill. 
Fernández Enguita, M. (1996). Escuela y Etnicidad: El caso del pueblo gitano.

Granada: C.I.D.E. Laboratorio de Estudios Interculturales.

Freeman, D. (1989). Teacher Training, Development, and Decision Making: A Model of Teaching and Related Strategies for Language Teacher Education. TESOL Quarterly, 23(1), 27-45.

Hall, G. (2011). Exploring English Language Teaching Language in Action. Oxon: Routledge.

Halliday, M.A.K. \& Matthiessen, C. (1999). Construing experience through meaning: A language-based approach to cognition. London: Cassell.

—. (2004). An introduction to functional grammar. London: Arnold.

Halliday, M.A.K. (1998). El lenguaje como semiótica social. La interpretación social del lenguaje y del significado. Ciudad de México: FCE.

. M.A.K. (2003). On language and linguistics. Londres: Continuum.

Hecht, M., Warren, J., Jung, E. \& Krieger, J. (2005). The Communication Theory of Identity: Development, Theoretical Perspective, and Future Directions. En W. Gudykunst (Ed.), Theorizing about Intercultural Communication (pp. 257-278). California: Sage Publications.

Holliday, A. (2003). Social Autonomy: Addressing the Dangers of Culturism in TESOL. En D. Palfreyman and R. Smith (Eds.), Learner Autonomy Across Cultures: Language Education Perspectives (pp. 110-126). Basingstoke: Palgrave Macmillan.

Iglesias Casal, I. (2016). El perfil del profesor de español como lengua extranjera: dimensión formativa, dimensión técnica y dimensión afectivo-emocional. Studia Romanica Posnaniensia, 43(3), 67-83.

Instituto Cervantes. (2018). El español: una lengua viva. Informe 2018. Recuperado de https://cvc.cervantes.es/lengua/ espanol_lengua_viva/

Johnson, M. (2004). A Philosophy of Second Language Acquisition. New York: Vail Ballou Press.

Kolb, D. (1984). Experiential learning Experiences as the Course of Learning and Development. Englewood Cliffs, NJ: Prentice Hall.

Kozulin, A., Gindis, B., Ageyev, V. S., \& Miller, S. M. (2003). Vygotsky's Educational Theory in Cultural Context. Cambridge: Cambridge University Press. 
Krashen, S. (1982). Principles and Practice in Second Language Acquisition. Oxford: Pergamon.

Kumaravadivelu, B. (2006). Understanding Language Teaching: From Method to Postmethod. Mahwah, NJ: Routledge.

Larsen-Freeman, D. (2000). Techniques and Principles in Language Teaching. Oxford: Oxford University Press.

Little, D. (2004). Autonomy and Autonomous Learners. En M. Bryam (Ed.), Routledge Encyclopedia of Language Teaching and Learning (pp. 69-72). London: Routledge.

Medina, A. \& Mata F. S. (2002). Didáctica General. Madrid: Prentice Hall. Nunan, D. (1991). Language Teaching Methodology. New York: Prentice Hall. Orbe, M. \& Spellers, R. (2005). From the Margins to the Center: Utilizing Co-Cultural Theory in Diverse Contexts. En W. Gudykunst (Ed.), Theorizing about Intercultural Communication (pp. 178192). California: Sage Publications.

Pizarro, M. (2013). Nuevas tareas para el professor de español como lengua extranjera: la reflexion sobre su concepción de enseñanza. Porta Linguarum, 19, 165-178.

Prahbu, N. S. (1990). There is No Best Method - Why?. TESOL Quarterly, 24(2), 161-176.

Richards, J. \& Lockhart, C. (1996). Reflective teaching in second language classrooms. Cambridge: Cambridge University Press.

Rodríguez-Perez, N. (2012). Causas que intervienen en la motivación del alumno en la enseñanza-aprendizaje de idiomas: el pensamiento del profesor. Didáctica, lengua y literatura, 24, 381-409.

Schön, D. (1992). La formación de profesionales reflexivos. Hacia un nuevo diseño de la enseñanza y del aprendizaje de los profesionales. Madrid: Paidós.

Shulman, L. S. (1986). Those Who Understand: Knowledge Growth in Teaching. Educational Researcher, 15(2), 4-14.

L. S. (1988). The dangers of dichotomous thinking in education. En P. Grimmet and G. Erickson (Eds.), Reflection in teacher education (pp. 31-39). New York: Teachers College Press.

Swain, M. (1985). Communicative Competence: Some Roles of Comprehensive Input and Comprehensive Output in its Development. En S. Gass and C. Madden (Eds.), Input and 
Second Language Acquisition (pp. 235-256). Rowley, MA: Newbury House.

Torres Torres, A. (2013). Del castellano de “un pequeño rincón” al español internacional. Norma, Revista de estudios lingüisticos hispánicos, 3, 205-224.

Zeichner, K (1993). El maestro como profesional reflexivo. Cuadernos de pedagogía, 220, 29-44.

Zeichner, K. \& Liston, D. (2014). Reflective teaching, an introduction. London: Routledge. 